procedimento pode ser realizado com segurança em qualquer idade gestacional e o feto pode apresentar bradicardia transitória com resolução espontânea. Episódios embólicos podem ocorrer após reversão de fibrilação atrial. Está indicado uso de anticoagulante em doses terapêuticas por 3 semanas antes da cardioversão, e por no mínimo 4 semanas após ${ }^{10,11}$. A anestesia para a cardioversão elétrica pode ser realizada com propofol (risco B, vide capítulo 6), etomidato (maior incidência de mioclonia), midazolan (risco D) ou thiopental (risco D). Todos atravessam a placenta e o risco é descrito de acordo com o grau de sedação fetal por ocasião do parto ${ }^{12,13}$.

\title{
Referências
}

1. Grinberg M, Cardoso LF. Sucesso e satisfação: a visão clínica da valvotomia mitral percutânea. Arq Bras Cardiol. 1997; 68: 145-6.

2. De Souza JA, Martinez EE Jr, Ambrose JA, Alves CM, Born D, Buffolo E, et al. Percutaneous balloon mitral valvuloplasty in comparison with open mitral valve commissurotomy for mitral stenosis during pregnancy. J Am Coll Cardiol. 2001; 37: 900-3.

3. Avila WS, Andrade J, Born D, Lopes CMC. Terapêutica da estenose mitral durante a gravidez: tratamento clínico, cirurgia cardíaca e valvotomia. Rev Soc Cardiol Estado de Sao Paulo. 1997; 2: 318-23.

4. Arnoni AS, Andrade J, Falcão HCB, Souza SCS. Cirurgia cardíaca na gravidez. Rev Bras Cir Cardiovasc. 1986; 1: 14-9.

5. Pomerantzeff PMA, Benício A, Brandão CMA, Avila WS, Bueno PC, Grinberg M. Cirurgia valvar em gestantes: experiência em oito casos. Arq Bras Cardiol. 1998; 70: 403-8.

6. Born D, Massonetto JC, De Almeida PA, Moron AF, Buffolo E, Gomes WJ, et al. Cirurgia cardíaca com circulação extracorpórea em gestantes: análise da evolução materno-fetal. Arq Bras Cardiol. 1995; 64: 207-11.
7. Arnoni RT, Arnoni AS, Bonini RC, De Almeida AF, Neto CA, Dinkhuysen J], et al. Risk factors associated with cardiac surgery during pregnancy. Ann Thorac Surg. 2003; 76: 1605-8.

8. Boyle McC, $\mathrm{O}^{\prime}$ Donnell MJ, Pantridge JF. Mitral valvotomy and pregnancy. Br Heart J. $1964 ; 26: 337-42$.

9. Leyse R, Oftsun M, Dillard DH, Merendino KA. Congenital aortic stenosis in pregnancy corrected by extracorporeal circulation. JAMA. 1961; 176: 1009-12.

10. Leung $C Y$, Brodsky MA. Cardiac arrhythmias and pregnancy. In: Elkayam U, Gleicher N. (editors.). Cardiac problems in pregnancy. New York: Wiley-Liss; 1998. p. 155-74.

11. Albers GW, DalenJE, LaupacisA, ManningWJ, Petersen P, SingerDE. Antithrombotic therapy in atrial fibrillation. Chest. 2001; 119 (Suppl): 194S-206S.

12. Briggs GG, Freeman RK, Sumner JY. (editors.). Drugs in pregnancy and lactation. 6th ed. Philadelphia: Lippincott Williams \& Wilkins; 2002.

13. Hullander RM, Leivers D, Wingler K. A comparison of propofol and etomidate forcardioversion. Anesth Analg. 1993; 77: 690-4.

\section{Assistência pré-natal obstétrica}

Clóvis Antônio Bacha, Frederico José Amedee Péret

No acompanhamento pré-natal, deve-se constituir uma equipe médica composta de obstetras, cardiologistas, anestesiologistas e pediatras treinados em cuidados com gestantes portadoras de doença cardiovascular. Idealmente, a paciente deve ser encaminhada a um centro de cuidados terciários.

As pacientes com lesões cardíacas graves e com alto risco de morte durante a gestação são candidatas ao abortamento terapêutico, que deverá ser sempre discutido considerando a vontade da paciente. O abortamento só se justifica em uma idade gestacional (IG) inferior a 20 semanas, período após o qual é preferível tentar atingir no mínimo a viabilidade fetal, já que a interrupção terapêutica passa a ter um risco semelhante ao de se continuar com a gestação ${ }^{1,2}$.

Condutas preconizadas:

1) Prevenção e controle de infecções - Deve ser realizada a imunização contra viroses antes da concepção (rubéola, hepatite $B$, influenza). Devem ser pesquisadas as infecções urinárias periodicamente, mesmo sem sintomatologia, e tratadas as infecções bacterianas com rigor. Atualmente é recomendável, também para gestantes normais, a pesquisa rotineira, entre 34 e 36 semanas de IG, da colonização em introito vaginal e retal pelo streptococcus do grupo B, agente frequente das pneumonias dos recém-nascidos, e de infecções puerperais ${ }^{3}$ (IB).

2) Inibição do trabalho de parto prematuro - Primeiramente deverá ser distinguido do falso trabalho de parto, adotando a conduta inicial de observação e repouso. O trabalho de parto não deverá ser inibido nas pacientes com cardiopatia descompensada (IC). O tratamento da descompensação cardíaca, com consequente melhora da perfusão tissular, frequentemente faz com que o trabalho de parto prematuro seja interrompido. Após a compensação, permanecendo a paciente com contrações, podem ser utilizados como tocolíticos a indometacina ou atosiban (IB). Essas drogas são as mais adequadas para portadoras de cardiopatia, hipertensão, diabetes e hipertireoidismo ${ }^{4}$. A indometacina, na dose inicial de 50 a $100 \mathrm{mg}$, seguida de $25 \mathrm{mg}$ via retal de $8 / 8 \mathrm{~h}$, deve ser restrita a gestações inferiores a 32 semanas e por período inferior a 48 horas (risco de fechamento precoce do ducto arteriovenoso, enterocolite, hemorragia intraventricular e oligohidrâmnio). O sulfato de magnésio não tem sido mais indicado como uterolítico (IIIA). Uma recente metanálise demonstrou sua ineficácia em retardar o parto pré-termo no período de 48 horas, além de aumentar a mortalidade perinatal em cerca de 2,8 vezes $^{5}$.

O uso de corticoide, para indução da maturidade pulmonar fetal e prevenção de hemorragia intracraniana, está indicado mesmo em pacientes descompensadas, desde 
que em idade gestacional entre 24 e 34 semanas e com perspectiva de parto num período de 24 horas a 7 dias. (IA) A retenção hídrica poderá ser combatida com uma dose maior de diuréticos (IlaC).

A corticoterapia consiste em: betametasona em duas doses IM de $12 \mathrm{mg}$, com intervalo de 24 horas, ou dexametazona em 4 doses IM de 6 mg de 12/12 ${ }^{6}$ (IA).

3) Condutas no trabalho de parto - Deve-se permitir que a paciente entre em trabalho de parto espontâneo ao termo e, com raras exceções, reservar a cesariana para as indicações obstétricas. Entretanto, nas pacientes com comprometimento da aorta com possível formação aneurismática (estenose e coartação de aorta, síndrome de Takayasu e Marfan), a cesárea é preconizada mediante anestesia raquidiana ou peridural, devido ao risco de rotura da aorta durante o trabalho de parto (IC). Recentemente tem-se utilizado óvulos com gel de prostaglandinas, com o objetivo de "amadurecer" o colo uterino desfavorável à indução do parto, conseguindo-se com isso minimizar o tempo decorrido entre o termo e o início do trabalho de parto em pacientes com lesões cardíacas (IB). O fórcipe de alívio deve ser utilizado no encurtamento do segundo período do trabalho de parto e na diminuição do esforço materno, minimizando as alterações cardiovasculares próprias deste estágio (IC).

Nas pacientes com cardiopatia grave e com repercussões fetais, as condições do concepto devem ser monitoradas continuamente (IC).

A paciente portadora de cardiopatia grave, que tolera adequadamente o trabalho de parto, com indicação de esterilização definitiva, tem no período de puerpério imediato a oportunidade adequada para ser submetida à salpingotripsia bilateral por incisão infraumbilical. Sempre devemos lembrar que os riscos de uma ligadura de trompas no puerpério são muito inferiores aos de uma nova gestação para esse grupo de pacientes (IlbC).

4) Controle das perdas sanguíneas - A ocitocina (10 unidades em $500 \mathrm{ml}$ de soro glicosado isotônico) e a curetagem pósparto devem ser empregadas quando necessário, com o objetivo de minimizar as perdas sanguíneas. Os ergotínicos devem ser evitados porque podem produzir elevações na pressão venosa central e hipertensão arterial transitória (IC).

5) Controle no pós-parto - A deambulação precoce e a amamentação devem ser incentivadas, a não ser que haja contraindicações. Nas indicações de inibição da lactação (CF IV NYHA e uso de amiodarona), recomenda-se enfaixamento das mamas e bolsa de gelo. O uso de drogas, como a bromocriptina $\left(\right.$ Parlodel $\left.^{\circledR}\right)$, é desaconselhado porque está associado a complicações, como vasoespasmo, hipertensão arterial, AVC isquêmico e hemorrágico, IAM, arritmias, convulsões e psicose puerperal ${ }^{7}$ (IIIB).

O acompanhamento por um período maior no puerpério é indicado nas pacientes portadoras de cardiopatias, devido ao risco acentuado de uma descompensação cardíaca e fenômenos tromboembólicos (IC).

Minimizar a quantidade de líquido administrado e indicar soroterapia só no pós-operatório de cesárea (IC). A analgesia pode ser realizada com uso de diclofenaco de sódio, e devido ao seu efeito antidiurético, deverá ser utilizado apenas nos dois primeiros dias de pós-parto, sendo posteriormente substituído por hioscina, dipirona e/ou paracetamol (IlaC). Sempre oferecer encaminhamento para avaliação e controle com equipe de planejamento familiar ${ }^{8}$ (IC).

6) Repercussões da gestação sobre o feto e o recémnascido - Vários fatores levam a uma maior morbimortalidade perinatal. Entre eles, a hipóxia, a utilização de medicamentos, a hereditariedade de certas cardiopatias e a possibilidade de infecção fetal pelo Trypanossoma cruzi. Esses fatores acarretarão, entre outras possíveis repercussões, uma maior incidência de prematuridade, de recém-nascidos pequenos para a idade gestacional, de abortamentos e de anomalias cardíacas e não cardíacas. Caso um dos pais tenha uma cardiopatia congênita, o recém-nascido tem uma chance de até $15 \%$ de ter uma anormalidade semelhante, aumentando para 50\% quando a anomalia é autossômica dominante, como ocorre na estenose subaórtica hipertrófica idiopática ou na síndrome de Marfan².

O desenvolvimento de episódios de descompensação cardíaca durante a gestação pode acarretar um pior prognóstico perinatal. Dentre as possíveis repercussões, está descrita uma maior incidência de baixo peso, prematuridade e índice de Apgar $<7$, além de um peso médio menor em 300 g no grupo de conceptos cujas mães desenvolveram complicações clínicas secundárias à cardiopatia, em relação àqueles cujas gestações não apresentaram tais complicações ${ }^{9}$.

A cianose materna aumenta o risco fetal, embora em formas cianóticas de doença cardíaca congênita existam certas respostas que servem para facilitar o recebimento de oxigênio pelo feto ${ }^{10,11}$. Apesar desse mecanismo de compensação, a maioria dos recém-nascidos de mães com cianose apresentam-se pequenos para a idade gestacional e/ou prematuros. Observa-se também uma elevada frequência de abortos, cuja incidência aumenta em paralelo com o hematócrito materno ${ }^{1}$.

Em pacientes transfundidas anteriormente, é essencial a pesquisa de infecções por hepatite $\mathrm{B}$ e pelo HIV, já que a utilização de medicamentos pode diminuir a transmissão vertical $^{12}(\mathrm{IA})$.

7) Rotina básica da avaliação fetal por ultrassonografia - Na gestação normal, existe uma rotina de avaliação ultrassonográfica fetal que consiste em:

\section{A) Avaliação no $1^{\circ}$ trimestre por via transabdominal ou} transvaginal:

- Confirmar a gestação (com 7 semanas);

- Estimar a idade gestacional;

- Diagnosticar gestações múltiplas.

B) Avaliação no $2^{\circ}$ e $3^{\circ}$ trimestres:

- Translucência nucal (com 10-14 semanas, auxilia no diagnóstico da síndrome de Down);

- Apresentação fetal;

- Volume de líquido amniótico;

- Atividade cardíaca fetal;

- Posição da placenta;

- Biometria fetal; 


\section{Diretrizes}

- Anatomia fetal (após as 18 semanas de IG);

- Estimativa de peso fetal.

Se for detectada alguma anormalidade, a avaliação deve ser complementada por exames mais específicos, como Doppler fetal, perfil biofísico fetal e ecocardiograma fetal ${ }^{13,14}$.

O ecocardiograma fetal deve ser realizado rotineiramente e sempre após as 20 semanas, por indicação materna ou fetal. As indicações maternas são: diabetes, cardiopatia congênita de um dos pais, infecção materna relacionada à teratogenicidade (rubéola, citomegalovírus, HIV), doença de Chagas e toxoplasmose (relacionadas a miocardiopatias ou miocardites fetais), idade materna $>35$ anos, fenilcetonúria, doenças do tecido conjuntivo (mais relacionadas com bloqueio atrioventricular fetal) e exposição a agentes teratogênicos. As indicações fetais são: achado de outras anormalidades fetais em estudo morfológico, doenças cromossômicas e arritmias fetais ${ }^{15,16}$. Deve ser considerado que a ultrassonografia nem sempre detecta todas as anomalias fetais e o diagnóstico definitivo pode ser obtido somente após o nascimento.

A periodicidade dos exames é decidida pelo obstetra, de acordo com a gravidade dos casos e o parâmetro a ser avaliado. Portadoras de cardiopatia cianótica ou com alterações hemodinâmicas importantes podem necessitar de reavaliação fetal por ultrassom, até mesmo semanal. O método auxilia também nos diagnósticos de morte fetal e descolamento prematuro de placenta.

\section{Referências}

1. Perloff JK. Gravidez e doenças cardiovasculares. In: Branwald E. Tratado de medicina cardiovascular. 2ª ed. São Paulo: Roca; 1987. p. 1726.

2. McAnulty JH, Metcalfe J, Ueland K. Cardiopatia e gravidez. In: Hurst JW. O coração. 6ª ed. Rio de Janeiro: Guanabara; 1990. p. 999.

3. Scharg S, Gorawitz R, Fultz-Butts K, Schuchart A. Prevention of perinatal group B streptococcal disease: revised guidelines from CDC MMWR Recomm Rep. 2002; 51 (RR-11): 1-22.

4. Scottish Obstetric Guidelines and Audit Project. (SOGAP), Sign Guideline "The management of postpartum haemorrhage". March, 2002.

5. Crowther CA, Hiller JE, Doyle LW. Magnesium sulphate for preventing preterm birth in threatened labour (Cochrane Review). In: The Cochrane Library, 4th ed. 2002.

6. Crowley P. Prophylatic corticosteroides for preterm birth (Cochrane Review). In: The Cochrane Library, 2002.

7. Iffy L, O'Donnell J, Correia J, Hopp L. Severe cardiac dysrhythmia in patients using bromocriptine postpartum. Am J Ther. 1998; 5: 111-5.

8. Bacha CA, Rezende CAL. Visão crítica na pesquisa dos fatores de risco para o desenvolvimento de complicações secundárias à cardiopatia materna. J Bras Ginec. 1998; 108: 51-97.

9. Bacha CA. Avaliação dos fatores de risco para o desenvolvimento de

\section{Fármacos cardiovasculares na gestação e amamentação}

\section{Maria Hebe Nóbrega de Oliveira, Maria Elizabeth Navegantes Caetano Costa, Paulo Roberto Pereira Toscano, Citânia Lúcia Tedoldi}

O maior problema terapêutico durante a gestação é o efeito adverso sobre o feto. O potencial efeito teratogênico é maior durante a embriogênese, que compreende as primeiras 8 semanas após a concepção. Entretanto, outros efeitos adversos podem ocorrer nos demais períodos da gestação. A Food and Drug Administration (FDA) classifica as drogas, considerando o risco para o feto, nas seguintes categorias:

$\mathbf{A} \rightarrow$ Estudos controlados em mulheres não demonstram risco para o feto no primeiro trimestre, não havendo evidência de risco nos demais; complicações clínicas secundárias à cardiopatia na gestante e análise de suas repercussões sobre a morbimortalidade materna e perinatal [dissertação]. Belo Horizonte: Faculdade de Medicina da UFMG; 1996.

10. Novy MJ, Peterson EN, Metcalfe J. Respiratory characteristics of maternal and fetal blood in cyanotic congenital heart disease. Am J Obstet Gynecol. 1968; 100: 821.

11. Whittemore R, Hobbins JC, Engle MA. Pregnancy and its outcome in women with and without surgical treatment of congenital heart disease. Am J Cardiol. 1982; $50: 641$.

12. Brocklehurst $P$, Volmink J. Antiretrovirals for reducing the risk of mothertochild transmission of HIV infection. The Cochrane Library, 2002.

13. Garra B, Meilstrup J. AIUM Prac tice Guideline for the Performance of an Antepartum Obstetric Ultrasound Examination. J Ultrasound Med. 2003; 22: $1116-25$.

14. Ultrasound screening for fetal abnormalities. Report of the RCOG Working Party. Supplement. London (UK); July 2000.

15. Zielinsky P. Abordagem diagnóstica e terapêutica pré-natal das anormalidades cardíacas fetais. Rev Bras Ecocardiogr. 1992; 17: 10-25.

16. Zielinsky P. Distúrbios do ritmo cardíaco fetal: detecção de conduta pré-natal. Arq Bras Cardiol. 1996; 66 (2): 83-6.

B $\rightarrow$ Estudos em animais não demonstraram risco fetal e não existem estudos controlados em mulheres no primeiro trimestre, não havendo evidência de risco nos demais;

$\mathbf{C} \rightarrow$ Estudos em animais não revelaram risco fetal, mas não há estudos controlados em mulheres nem em animais, e a droga deve ser administrada quando o risco potencial justifica o benefício;

D $\rightarrow$ Há evidência de risco fetal em humanos, mas os benefícios são aceitáveis, apesar dos riscos;

$\mathbf{X} \rightarrow$ Estudos em animais e humanos demonstraram anormalidades fetais, sendo a droga contraindicada em mulheres que estão ou querem se tornar gestantes.

No momento do aleitamento, a farmacocinética das drogas no leite materno depende de vários fatores e o parecer em relação à segurança na amamentação é orientado pela Academia Americana de Pediatria 